\title{
Fundamental theory of void fraction of cohesive spheres with size distribution and its application to multi component mixture system
}

\author{
Yutaka AIKAWA, ${ }^{\dagger}$ Maki INOUE and Etsuo SAKAI* \\ General R\&D Laboratories, Taiyo Yuden Co, Ltd., 5607-2, Nakamuroda-machi, Takasaki, Gunma 370-3347, Japan \\ *Department of Metallurgy and Ceramics Science Graduate, Graduate School of Science and Engineering, \\ Tokyo Institute of Technology, 2-12-1 Ookayama, Meguro-ku, Tokyo, Japan
}

\begin{abstract}
In this paper, a fundamental theory of packing density for particles with different size distributions is derived using a statistical method. The use of this theory enables calculation of the packing density of a particle system because the packing density depends on the size distribution function. This theory can also be applied to the calculation of the density of a multi-component mixture system by considering its size distribution function.
\end{abstract}

(02012 The Ceramic Society of Japan. All rights reserved.

Key-words : Packing density, Void fraction, Size distribution, Statistical method, Coordinate number, Sphere, Mixture system

[Received August 3, 2011; Accepted October 18, 2011]

\section{Introduction}

In recent times, ceramic capacitors, which are of considerable practical importance in various electronic devices, have been developed. Furthermore, several ferroelectric substances are used for manufacturing ceramic capacitors such as thin-film capacitors, multilayer ceramic capacitors, and those used in other dielectric applications. ${ }^{1)}$

In order to optimize the properties of such devices, it is necessary to analyze optimum conditions associated with the ferroelectric properties of ceramics. One of the methods for increasing the capacitance of ceramic capacitors is to increase the sintering density of ceramics by the decrease in the size of the internal pores. It is important to reduce the internal pore size of the raw material required in the construction of ceramics because the sintering density of ceramics is proportional to the packing state of the raw material. Thus, if the density of rare powder of the raw material or the mixture of raw materials can be estimated theoretically, it would be possible to identify the optimal conditions for the sintering of ceramics.

In order to analyze the packing state of materials, many fundamental theories were developed and calculations were performed in the context of fundamental systems used for sphere packing. As a fundamental problem, the random packing density of equal spheres was calculated by computer simulations. ${ }^{2)}$ Further, random packed structures were analyzed in terms of the radial distribution function, ${ }^{3)}$ and the results were compared with those of the analysis of dense random packed structures. ${ }^{4), 5)}$

In general, the calculation of powder density encounters some problems resulting from particle shape, ${ }^{6), 7)}$ interaction among particles, ${ }^{8,9)}$ and so on. Although several attempts have been made to solve these problems, only the calculation of dense packing that deals with a ternary mixture of spheres has been done successfully. ${ }^{10)-12)}$ Moreover, the effects of multi-compo-

$\uparrow$ Corresponding author: Y. Aikawa; E-mail: y-aikawa@jty.yuden. co.jp nent particle mixture systems ${ }^{13)-16)}$ and size distribution ${ }^{17), 18)}$ on packing density have been investigated. The most important topic in the field of sphere packing is assumed to be the fundamental theory of packing density with a size distribution function. In addition, the effects of particle shape, interaction among particles, and so on, may also be considered on the basis of this fundamental theory.

M. Suzuki et al. investigated the void fraction in a cohesivesphere system consisting of maximum three kinds of spheres with different radii by using an empirical formula and by performing computer simulations, and the estimated results almost agree with the experimental results. ${ }^{19)}$ Taking into consideration by using a modified version of the method of M. Suzuki, ${ }^{20), 21)}$ we formulated the fundamental theory of the void fraction with size distribution based on three-dimensional geometry and a statistical method.

\section{The theory of void fraction of spherical-particle system with size distribution}

In order to deal with a cohesive-particle system, for simplicity, the shape of particles is assumed to be spherical. The distribution function of a system of spherical particles, $f\left(r_{i}\right)$, as shown in Fig. 1 is normalized as

$$
\sum_{i} f\left(r_{i}\right)=1,
$$

where $r_{i}$ is the radius of the $i$-th particle. In order to analyze the void fraction of the particle system, we first consider a sphere of radius $R$ ( $R$-sphere), which is enclosed in direct contact with spheres of radius $r$ ( $r$-spheres), as shown in Fig. 2. It is assumed that a hypothetical sphere with radius $\sqrt{(r+R)^{2}-r^{2}}$ encloses an $R$-sphere in its core. We introduce the total volume of the void, $\Delta V_{R r}$, which exists between the surface of the $R$-sphere and the surface of the hypothetical sphere. The total void volume $\Delta V_{R r}$ is expressed in terms of the total void volume $\Delta V_{R r}{ }^{(1)}$ and $\Delta V_{R r}{ }^{(2)}$. Here, $\Delta V_{R r}{ }^{(1)}$ exists between the projection of an $r$-sphere on the $R$-sphere and the hypothetical sphere within the solid angle $\Omega$, 


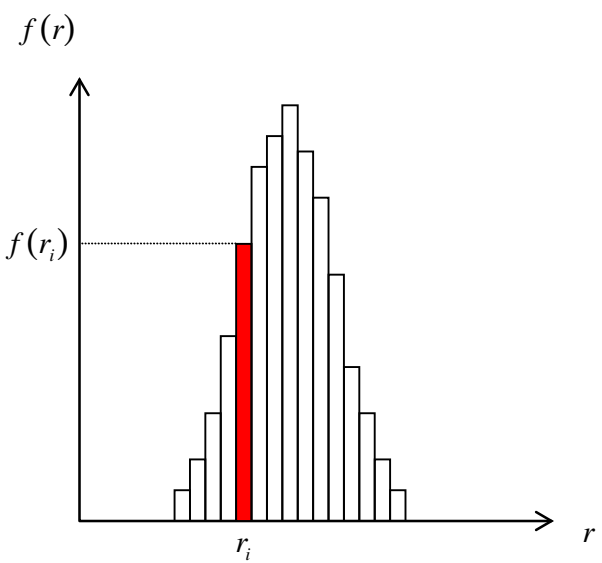

Fig. 1. (Color online) Example of a size distribution function.

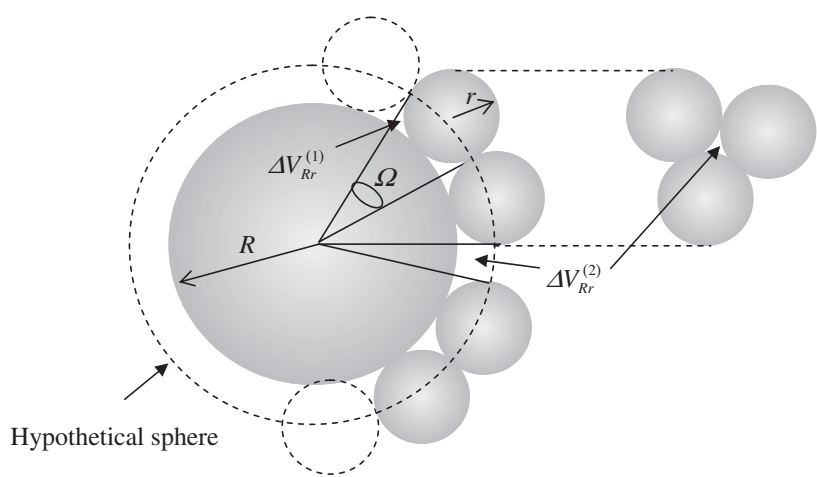

Fig. 2. Packing of spheres with radius $r$ in direct contact with sphere with radius $R$.

and $\Delta V_{R r}{ }^{(2)}$ exists between the projection of the total open area on the $R$-sphere and the hypothetical sphere within the total solid angle of the total open area:

$$
\Delta V_{R r}=\Delta V_{R r}{ }^{(1)}+\Delta V_{R r}{ }^{(2)} .
$$

Thus, the void fraction within the radius of the hypothetical sphere enclosed by $r$-spheres is given by

$$
\delta_{R r}=\frac{\Delta V_{R r}}{V},
$$

where, $V$ is the volume of the hypothetical sphere. In the case of a size distribution system consisting of $N$ spherical particles, the number of particles with radius $r_{j}$ ( $j$-particles), $n_{j}$, is related to the distribution function $f\left(r_{i}\right)$ of the system as

$$
\frac{n_{j}}{N}=f\left(r_{j}\right)
$$

Here, the left-hand side of the equation is the occupancy rate of $j$-particles. The occupancy ratio of the surface area of the $i$-particles having the same radius $r_{i}$ is represented by $S_{i} / S$, where $S$ is the total surface area of the all particles in the system, and $S_{i}$ is the total surface area of the $i$-particles. The coordination number $N_{j}$ around a $j$-particle is given by

$$
N_{j}=\sum_{i} \frac{S_{i}}{S} N_{j i}(\max )
$$

Here, $N_{j i}(\max )$ is defined as the maximum number of $i$-particles which enclose with the $j$-particle in direct contact. In the case of spherical particle system, $S_{i}$ is given as

$$
S_{i}=4 \pi r_{i}^{2} f\left(r_{i}\right) .
$$

By using the coordination number $N_{j}$ in Eq. (5), the average coordination number is given by

$$
N=\sum_{j} f\left(r_{j}\right) N_{j}
$$

or

$$
N=\sum_{i} \sum_{j} \frac{r_{i}^{2} f\left(r_{i}\right) f\left(r_{j}\right)}{\left\langle r^{2}\right\rangle} N_{j i}(\max )
$$

The packing rate of the hypothetical sphere of a $j$-particle, which is surrounded by densely packed $i$-particles, is expressed using Eq. (3) as

$$
p_{j i}(\max )=1-\delta_{j i}(\min ) .
$$

The packing ratio of a $j$-particle is given by

$$
p_{j}=\sum_{i} \frac{S_{i}}{S} p_{j i}(\max ),
$$

where $p_{j i}(\max )$ is defined as the maximum packing ratio of $i$-particles having radius $r_{i}$ around the $j$-particles. The occupancy ratio of the volume of the $j$-particles is represented by $V_{j} / V$, where $V$ is the total volume of the entire system, and $V_{j}$ is the total volume of particles with the same radius $r_{j}$. The average packing ratio is given by

$$
p=\sum_{j} \frac{V_{j}}{V} p_{j}
$$

In the case of a spherical-particle system, $V_{j}$ is given as

$$
V_{j}=\frac{4}{3} \pi r_{j}^{3} f\left(r_{j}\right)
$$

Consequently, the packing ratio is expressed as

$$
p=\sum_{i} \sum_{j} \frac{r_{i}^{2} r_{j}^{3} f\left(r_{i}\right) f\left(r_{j}\right)}{\left\langle r^{2}\right\rangle\left\langle r^{3}\right\rangle} p_{j i}(\max ) .
$$

Thus, the void fraction is given by

$$
\delta=1-p
$$

By calculating $\Delta V_{j i}$ in Eq. (2) and by performing a geometrical analysis or numerical calculation using computer simulations, we can calculate the void fraction given by Eq. (11) for a sphericalparticle system with any arbitrary size distribution $f(r)$.

\section{Comparison between experimental and theoretical results}

As an example the experimental data of the packing ratio for the two types of particles, $i$ - and $j$-particles, with a radius ratio $r_{i}: r_{j}$ of 1:5 are plotted in Fig. 3. The horizontal axis represents the volume ratio of the two types of particles. The respective particle systems were immersed in separate liquids: the volumes of those liquids were measured to determine the void fractions, and then, the actual measured values were determined on the basis of the obtained void fractions. It is obvious that the theoretical calculation results (curved line) approximately corresponded to the experimental results (rhombic dots) both qualitatively and quantitatively. Figure 4 shows a simple system with just one type of particle $(5 \mathrm{~mm} \varphi)$ and a system with two types of particles with different radii $(1$ and $5 \mathrm{~mm} \varphi)$ in the ratio 3:7. The ratio $3: 7$ provides the highest packing rate in our experiments. 


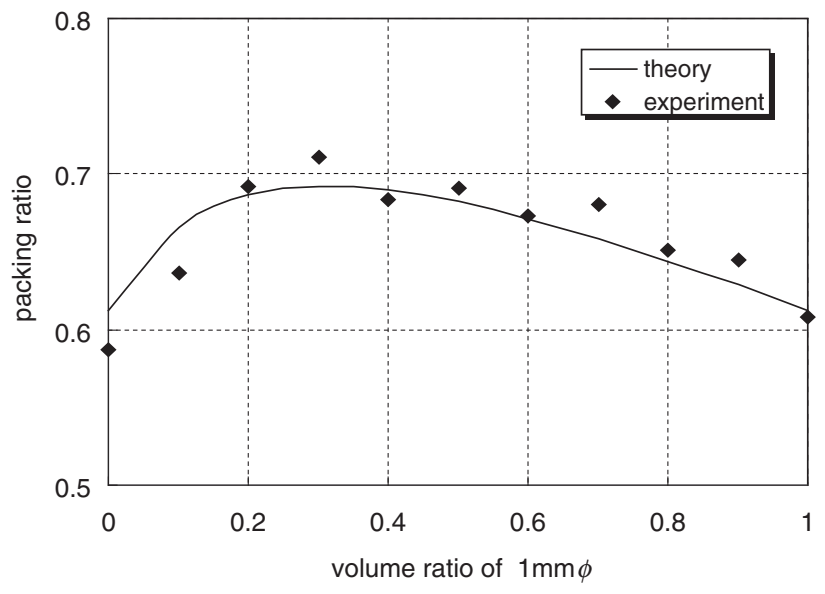

Fig. 3. Packing density of mixed-particle system comprising particles with two different radii. The horizontal axis represents the volume ratio of particle diameter of $1 \mathrm{~mm} \varphi$.

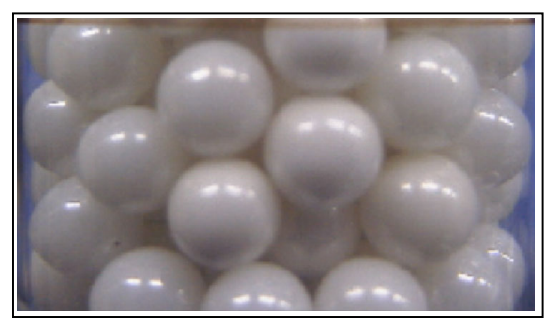

Simple system consisting of single type of particles

$(5 \mathrm{~mm} \phi)$

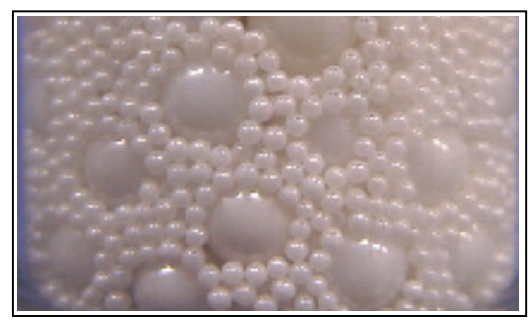

System with particles having two different diameters particles

$(1 \mathrm{~mm} \phi: 5 \mathrm{~mm} \phi=3: 7)$

Fig. 4. (Color online) Mixed-particle system consisting of particles with diameters of 1 and $5 \mathrm{~mm} \varphi$.

\section{Mixed system having different size distributions}

On the basis of our theory, we consider the packing rate of a system consisting of different particle systems with the size distribution functions $f_{1}\left(r_{i}\right), f_{2}\left(r_{i}\right), \ldots$ We consider a system with size distribution $f_{j}\left(r_{i}\right)$, volume $V_{j}$, and packing ratio as $p_{j}$. The number of particles, $N_{j}$, in this system is given by

$$
N_{j}=\frac{p_{j} V_{j}}{\frac{4 \pi}{3} \sum_{k} r_{k}^{3} f_{j}\left(r_{k}\right)},
$$

where the denominator is the average volume of the particles in this system.

The number of particles of radius $r_{i}$ in the system with size distribution $f_{j}\left(r_{i}\right)$ is

$$
N_{j}\left(r_{i}\right)=N_{j} f_{j}\left(r_{i}\right)=\frac{p_{j} V_{j} f_{j}\left(r_{i}\right)}{\frac{4 \pi}{3} \sum_{k} r_{k}^{3} f_{j}\left(r_{k}\right)} .
$$

Therefore, the size distribution of the mixture system consisting of multiple systems with size distributions $f_{j}\left(r_{i}\right)(j=1,2, \ldots)$ is given by

$$
F\left(r_{i}\right)=\frac{\sum_{j} N_{j}\left(r_{i}\right)}{\sum_{j} N_{j}}=\sum_{j} \frac{p_{j} V_{j} f_{j}\left(r_{i}\right)}{\sum_{k} r_{k}^{3} f_{j}\left(r_{k}\right)} / \sum_{j} \frac{p_{j} V_{j}}{\sum_{k} r_{k}^{3} f_{j}\left(r_{k}\right)} .
$$

In order to calculate the packing ratio of the mixture system, $f\left(r_{i}\right)$ in Eq. (10) should be replaced with $F\left(r_{i}\right)$.

By applying this theory to the data of the size distribution of the two types of raw materials, as shown in Fig. 5, we can determine the optimum conditions for obtaining the highest packing ratio of mixed powder of these materials. For example, we try to calculate the packing ratio of this mixed powder having different mixing ratios of the two types of raw materials $\mathrm{A}$ and $\mathrm{B}$, as shown in Fig. 5. First, the size distribution $F(r)$ of the mixed powder for different mixing ratios is derived from Eq. (14); second, the packing ratio is calculated by Eq. (10) using each size distribution $F(r)$ for each mixing ratio. As shown in Fig. 6 the solid curve is the theoretical packing ratio of the mixed powder system of materials A and B. In this case, the optimum condition that would give the highest packing ratio is the A:B mass ratio of 0.26:0.74. It is confirmed that sintering density becomes maximum under this optimum condition. The measured packing ratio of the mixed materials is shown by the cubic dots (mean values) in Fig. 6. In this experiment, the samples were prepared in the form of thin films. The liquid membrane of the solution, which was a dispersion system of the mixed powders in an organic solvent, was prepared on a plate by spin coating. The thin films were prepared by drying the liquid membrane, and they were posttreated in a furnace maintained at an appropriate temperature. The thickness of the thin films was about $200 \mathrm{~nm}$. In Fig. 6, the solid line represents the values calculated by the present theory, which clearly reveals that the actual measured values were qualitative and quantitative agreement with the theoretical calculation values.

\section{Conclusion}

In order to reduce the size of internal pores of sintered ceramics and to enhance the properties of ceramic devices, it is important

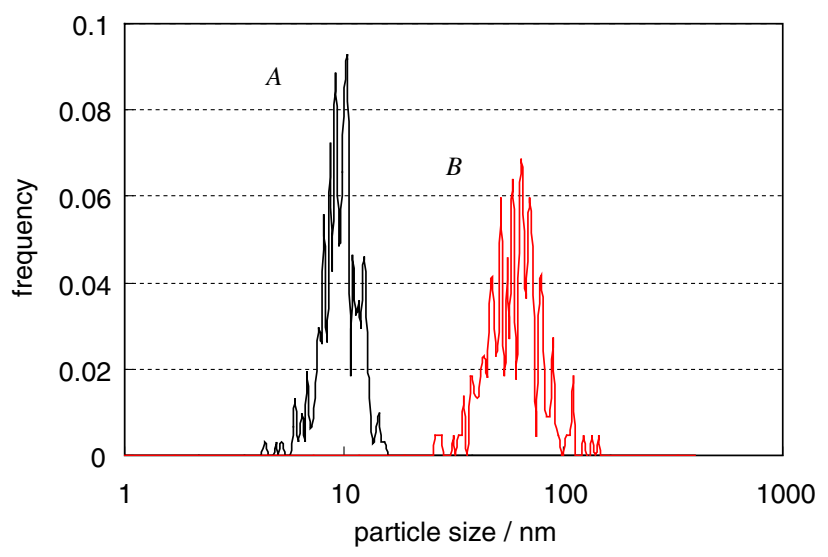

Fig. 5. (Color online) Experimental data of size distribution of raw materials $\mathrm{A}$ and $\mathrm{B}$. 


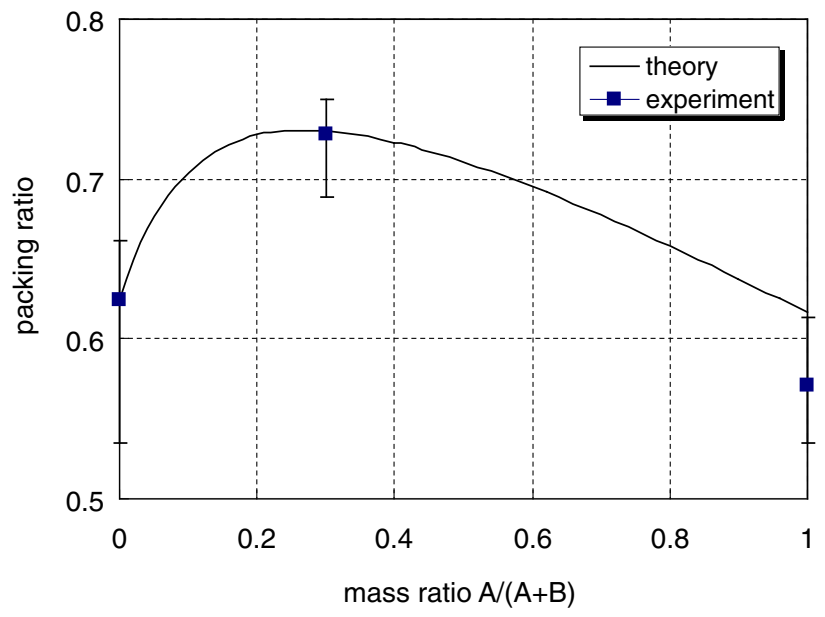

Fig. 6. (Color online) Comparison between theoretical and experimental values of the mixed powder system consisting of materials $\mathrm{A}$ and $\mathrm{B}$ shown in Fig. 5.

to identify the optimum condition that would give the highest packing ratio of raw materials. Although the presence of nonuniform sized particles in materials poses several difficulties in the analysis of the above problem, it is possible to calculate the packing ratio of materials having arbitrary size distributions and mixture systems of these materials by using our proposed theory. The use of this theory would help in achieving the optimum ceramic properties; it would also lead to reduction of effort and time required for identifying the optimum conditions in the field of rheology.

Acknowledgements The author would like to thank Mrs. Fumi Nakasone of Taiyo Yuden Co., Ltd., assistance with preparing samples and measurements. Further, the author would like to express his gratitude to Professor Michitaka Suzuki of the University of Hyogo for extensive discussions and valuable suggestions.

\section{References}

1) H. Kishi, Y. Mizuno and H. Chazono, Jpn. J. Appl. Phys., 42, $1-15(2003)$.

2) J. D. Bernal and J. L. Finney, Discuss. Faraday Soc., 43, 6269 (1967).

3) G. D. Scott and D. M. Kilgour, J. Phys. D: Appl. Phys., 2, 863866 (1969).

4) D. J. Adams and A. J. Matheson, Chem. Phys., 56, 1989-1994 (1972).

5) A. J. Matheson, J. Phys. C: Solid State Phys., 7, 2569-2576 (1974).

6) K. Takenaka, K. Iimura, M. Suzuki and M. Hirota, Adv. Powder Technol., 19, 25-37 (2008).

7) S. Liu and Z. Ha, Powder Technol., 126, 283-296 (2002).

8) D. Pinson, R. P. Zou, A. B. Yu, P. Zulli and M. J. McCarthy, J. Phys. D: Appl. Phys., 31, 457-462 (1998).

9) R. Y. Yang, R. P. Zou and A. B. Yu, Phys. Rev. E: Stat. Phys., Plasmas, Fluids, Relat. Interdiscip. Top., 62, 3900-3908 (2000).

10) J. A. Dodds, J. Colloid Interface Sci., 77, 317-327 (1980).

11) T. Stovall, F. Delarrard and M. Buil, Powder Technol., 48, 112 (1986).

12) A. B. Yu and N. Standish, Powder Technol., 55, 171-186 (1988).

13) J. S. Marshall and V. K. Dhir, Chem. Eng. Commun., 48, 261285 (1986).

14) R. P. Zou, C. L. Feng, J. Q. Xu and A. B. Yu, Ind. Eng. Chem. Res., 44, 8401-8408 (2005).

15) A. B. Yu and N. Standish, Ind. Eng. Chem. Res., 30, 1372$1385(1991)$.

16) A. B. Yu, J. Bridgwater and A. Burbidge, Powder Technol., 92, 185-194 (1997).

17) N. Ouchiyama and T. Tanaka, Ind. Eng. Chem. Fundam., 20, 66-71 (1981)

18) N. Ouchiyama and T. Tanaka, Ind. Eng. Chem. Fundam., 25, 125-129 (1986).

19) M. Suzuki and T. Oshima, Powder Technol., 36, 181-188 (1983).

20) M. Suzuki and T. Oshima, Powder Technol., 35, 159-166 (1983).

21) M. Suzuki, H. Kada and M. Hirota, Adv. Powder Technol., 10, 353-365 (1999). 